\title{
Interdisziplinär zur optimalen Diabetes-Behandlung
}

\author{
Die meisten Diabetologischen Notfälle bei Patienten zwischen 45 und 75 Jahren
}

Einem Bericht der WHO zufolge, leiden europaweit etwa 23 Millionen Erwachsene an Diabetes mellitus. In Österreich sind etwa 550.000 Menschen betroffen, wobei es ein West-Ost-Gefälle gibt mit einer größeren Häufigkeit im Osten und vor allem in der Großstadt Wien. Beim dritten Diabetes-Symposium des HerzJesu Krankenhauses in Wien wurden Ende Februar praxisnah und interdisziplinär die aktuellsten State-of-the-ArtTherapien in der Diabetesbehandlung sowie Maßnahmen in medizinischen Spezialsituationen wie zum Beispiel diabetologischen Notfällen.

Die Optimierung der Zusammenarbeit bei der Diabetesbehandlung bildet einen wesentlichen Bestandteil des Patientenmanagements. Dabei geht es um den fachli- chen Austausch und die Möglichkeit, bei konkreten Fragestellungen den jeweiligen Experten direkt zu Rate zu ziehen. „Eine gute Zusammenarbeit zwischen niedergelassenen Allgemeinmedizinern, Fachärzten, Diätologen und Diabetesberatern sorgt für eine verbesserte Betreuung der Diabetespatienten", stellte Doz. Dr. Edmund Cauza, Leiter der Abteilung für Innere Medizin fest. Der Patient erfährt so nicht nur eine bestmögliche Behandlung sondern auch eine umfassende Schulung und Aufklärung über Diabetes.

Diabetologische Notfälle sind nach wie vor häufig. Meist handelt es sich dabei um Hypoglykämie oder Ketoazidose. Bei der Hypogykämie sind zum Beispiel Zittern, Schweißausbrüche und im Verlauf auch Krämpfe typische Symptome, die bis zum Koma führen können. Die Hypoglykämie muss durch sofortige Zuckergabe behandelt werden. Ketoazidose tritt bei absolutem Insulinmangel auf und wird durch sofortige Insulinverabreichung behandelt. Als Symptome treten eine vertiefte KussmaulAtmung (erhöhte Atemtätigkeit) sowie Bewusstseinsstörungen ebenfalls bis zum Koma auf. Zwischen 1993 und 2005 suchten 34 von 1.000 Diabetikern eine Notfalleinrichtung auf. Besonders betroffen von diabetologischen Notfällen sind Patienten im Alter zwischen 45 und 75 Jahren.

Quelle: Presseinformation Herz-Jesu Krankenhaus

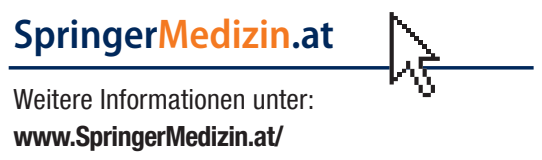

